\title{
RADIATIVE WINDS FROM ACCRETION DISKS IN CVS
}

\author{
M. HACHIYA, Y. TAJIMA, J. FUKUE \\ Astronomical Institute, Osaka Kyoiku University, \\ Asahigaoka, Kashiwara, Osaka 582, Japan
}

P Cyg profiles observed in CVs strongly suggest the existence of accretion disk winds in CVs. Recently, X-ray observations revealed bipolar outflows in supersoft $\mathrm{X}$-ray sources. The wind velocity measured in these observations is about $3000-5000 \mathrm{~km} \mathrm{~s}^{-1}$, which is of the order of the es cape velocity of white dwarfs. Hence, it is supposed that the winds would originate from the inner disk and/or the boundary layers between the disk and white dwarf. We examine radiatively-acclerated accretion-disk winds, and found that the terminal speed of the wind emanated from the inner region becomes $\sim 0.6 \sqrt{G M / r_{\text {in }}}$, where $M$ is the white dwarf mass and $r_{\text {in }}$ the inner radius of the disk.

\section{Radiation Fields of the Disk and the Boundary Layer}

For the present purpose, we quantitatively calculate radiation fields produced by the standard disk and the boudary layer. Examples of radiation fields are shown below, where the isoflux contours are displayed.
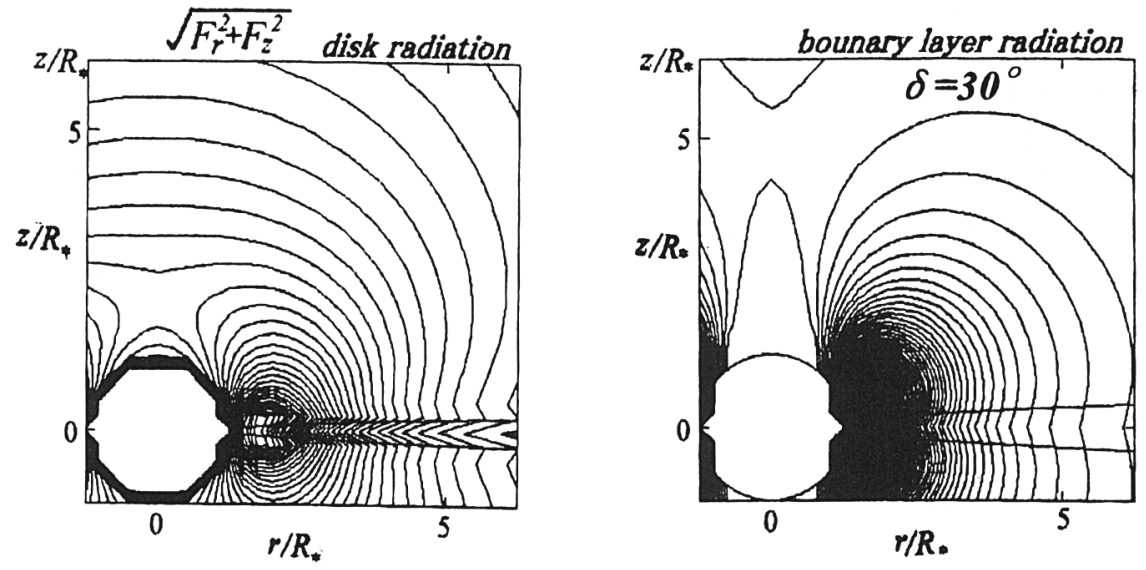


\section{Particle Winds}

Using the radiation fields obtained numerically, we calculate motions of the winds, which is assumed to consist of normal plasmas.

Trajectories of particle winds ejected from the disk surface are shown below (left panel). The abscissa is the distance from the central object and the ordinate is the height from the disk surface measured by a white dwarf radius $R_{\star}$.

Terminal velocities of radiatively-accelerated particle winds are also shown for various disk luminosities (right panel), where the normalized disk luminosity $\Gamma_{d}$ is set to be equal to the normalized star luminosity $\Gamma_{\star}$. The abscissa is the initial radii of the winds in units of $R_{\star}$, and the ordinate is the terminal velocity in units of the Keplerian rotation speed at $R_{\star}$, $G M / R_{\star}$. The normalized luminosities are attached on each curve.
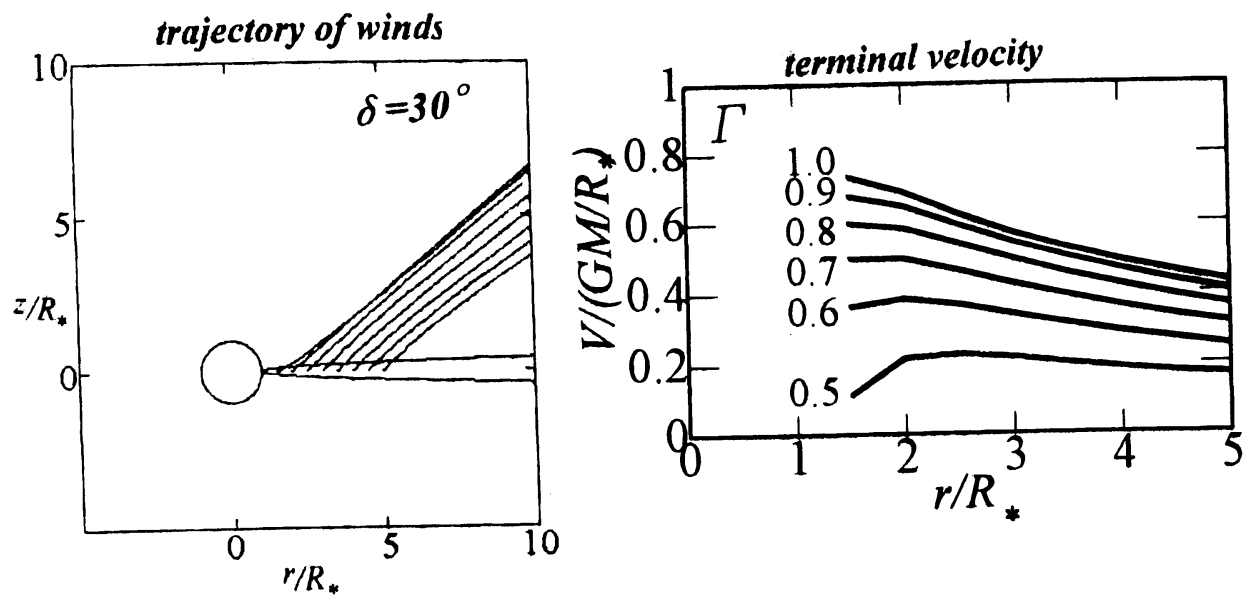

\section{Concluding Remarks}

We calculate the radiation fields produced by a standard accretion disk and a boundary layer, and the radiatively-accelerated accretion-disk winds, numerically. We found that the terminal speed of the wind emanated from the inner region becomes $\sim 0.6 \sqrt{G M / r_{\text {in }}}$, that is consistent with observations.

\section{Reference}

Tajima Y., Fukue J. 1996, PASJ 48, 529

Tajima Y., Fukue J. 1997, in this volume 